\title{
CD30 expression in neoplastic T cells of follicular T cell lymphoma is a helpful diagnostic tool in the differential diagnosis of Hodgkin lymphoma
}

\author{
Sylvia Hartmann ${ }^{1,2} \cdot$ Olga Goncharova $^{1} \cdot$ Anna Portyanko $^{3} \cdot$ Elena Sabattini $^{4} \cdot J_{\text {örn Meinel }}^{5} \cdot$ Ralf Küppers $^{6} \cdot$ \\ Claudio Agostinelli ${ }^{7}$ Stefano Aldo Pileri, ${ }^{7,8}$ Martin-Leo Hansmann ${ }^{1,2,8}$
}

Received: 5 May 2018 / Revised: 21 June 2018 / Accepted: 23 June 2018 / Published online: 23 August 2018

(c) United States \& Canadian Academy of Pathology 2018

\begin{abstract}
Follicular T cell lymphoma is derived from follicular T-helper cells. In many cases, neoplastic T cells form rosettes around Hodgkin-Reed-Sternberg-like cells, which can lead to the misdiagnosis of classical Hodgkin lymphoma. The aim of the present study was to obtain a better understanding of this rosetting phenomenon and to recognize features that are helpful in the differential diagnosis of classical Hodgkin lymphoma. Sixteen mostly elderly follicular T cell lymphoma patients (mean 66 years) were analyzed. Fifteen of the 16 follicular T cell lymphoma cases presented with Hodgkin-Reed-Sternberg-like cells, which were CD20-positive in $27 \%$ of the cases and Epstein-Barr virus-infected in nearly all cases. Frequently, the immunophenotype of rosetting neoplastic $\mathrm{T}$ cells differed from the bulk neoplastic cells with less numerous $\mathrm{T}$-follicular helper cell markers expressed, suggesting a modulation of T-follicular helper cell marker expression in the neoplastic $\mathrm{T}$ cells. In $75 \%$ of the cases, variable CD30 expression was encountered in the neoplastic T cells, likely reflecting an activation state in these cells. Hodgkin-Reed-Sternberg-like cells were positive for CCL17, and follicular T cell lymphoma tumor cells expressed its receptor CCR4 at variable intensity, thus potentially explaining the phenomenon of the tumor cells' rosetting around Hodgkin-Reed-Sternberg-like cells. In summary, this study confirms the presence of Hodgkin-Reed-Sternberg-like cells in a high number of cases of follicular $\mathrm{T}$ cell lymphoma, suggesting that Hodgkin-Reed-Sternberg-like cells may contribute to the development of this lymphoma. Hodgkin-Reed-Sternberg-like cells in follicular T cell lymphoma cannot reliably be differentiated from the Hodgkin-Reed-Sternberg cells of classical Hodgkin lymphoma based on their immunophenotype. In contrast, demonstration of a T-follicular helper cell phenotype with CD10 and frequent CD30 expression in the neoplastic $\mathrm{T}$ cell population can help to establish the diagnosis of follicular $\mathrm{T}$ cell lymphoma, and may even indicate CD30 as a therapeutic target for these patients.
\end{abstract}

Electronic supplementary material The online version of this article (https://doi.org/10.1038/s41379-018-0108-5) contains supplementary material, which is available to authorized users.

Sylvia Hartmann

s.hartmann@em.uni-frankfurt.de

1 Dr. Senckenberg Institute of Pathology, Goethe University Hospital, Frankfurt am Main, Germany

2 Reference and Consultant Center for Lymph node and Lymphoma pathology, Frankfurt am Main, Germany

3 NN Alexandrov National Cancer Center, Minsk, Belarus

4 Department of Diagnostic, Experimental and Specialty Medicine, Haematopathology Section, University of Bologna, S Orsola-

\section{Introduction}

Recently, the 2017 revision of the WHO Classification of Tumors of Haematopoietic and Lymphoid Tissues has recognized an overall category of nodal peripheral $\mathrm{T}$ cell lymphomas with $\mathrm{T}$-follicular helper cell origin [1]. This

Malpighi Hospital, Bologna, Italy

5 Department of Pathology, Carl Gustav Carus University Hospital, Technische Universität Dresden, Dresden, Germany

6 Institute of Cell Biology (Cancer Research), Faculty of Medicine, University of Duisburg-Essen, Essen, Germany

7 Haematopathology Unit, European Institue of Oncology, Milan, Italy

8 Frankfurt Institute of Advanced Studies, Frankfurt am Main, Germany 
category comprises angioimmunoblastic $\mathrm{T}$ cell lymphoma, follicular $\mathrm{T}$ cell lymphoma, and other nodal peripheral $\mathrm{T}$ cell lymphomas with $\mathrm{T}$-follicular helper cell phenotype. Angioimmunoblastic $\mathrm{T}$ cell lymphoma and other T-follicular helper cell-associated peripheral $\mathrm{T}$ cell lymphomas have common and distinct features. Genetic studies have shown that these groups of peripheral $\mathrm{T}$ cell lymphomas share the same mutational landscape originally reported in angioimmunoblastic T cell lymphoma [2]. To date, comprehensive molecular data on follicular $\mathrm{T}$ cell lymphoma are lacking. The translocation $\mathrm{t}(5 ; 9)(\mathrm{q} 33$; q22), involving the fusion of ITK (IL-2-inducible T cell kinase) and $S Y K$ (spleen tyrosine kinase), has been reported in approximately $20 \%$ of follicular $\mathrm{T}$ cell lymphoma cases [3, 4], and only occasionally in angioimmunoblastic T cell lymphoma [5]. All types of peripheral $\mathrm{T}$ cell lymphomas with $\mathrm{T}$-follicular helper cell phenotype can contain Hodgkin and Reed-Sternberg-like cells, which are in most cases Epstein-Barr virus (EBV)-positive and appear as a secondary effect to local immunosuppression [6, 7]. Follicular $\mathrm{T}$ cell lymphoma can be mistaken for classical Hodgkin lymphoma and a variety of other malignant lymphomas [8], as well as reactive changes due to the presence of Hodgkin-Reed-Sternberg-like cells, often partial infiltration of only the lymph node and, frequently, a small neoplastic clone. In contrast, the diagnosis of angioimmunoblastic $\mathrm{T}$ cell lymphoma and other nodal T-follicular helper cell-like peripheral $\mathrm{T}$ cell lymphomas is rather straightforward, despite the possible presence of Hodgkin-Reed-Sternberg-like cells.

Follicular $\mathrm{T}$ cell lymphoma can manifest in two main morphologic patterns: follicular lymphoma-like, which forms intrafollicular aggregates and can closely mimic follicular lymphoma, or progressively transformed germinal center-like, which, as the name implies, resembles progressively transformed germinal centers [3, 9]. The progressively transformed germinal center-like pattern is approximately threefold more frequently encountered than is the follicular lymphoma-like pattern [3].

The nodular or follicular growth pattern of follicular $\mathrm{T}$ cell lymphoma, as well as the presence of EBV-positive Hodgkin-Reed-Sternberg-like cells in this tumor, can lead to a misdiagnosis of nodular lymphocyte predominant Hodgkin lymphoma or lymphocyte-rich classical Hodgkin lymphoma. Apart from Hodgkin-ReedSternberg-like cells, another feature common to classical and nodular lymphocyte predominant Hodgkin lymphoma, as well as follicular $\mathrm{T}$ cell lymphoma, is the presence of rosetting $\mathrm{T}$ cells around Hodgkin-ReedSternberg (-like) cells. In classical and nodular lymphocyte predominant Hodgkin lymphoma, these rosetting
$\mathrm{T}$ cells are polyclonal [10], whereas in follicular $\mathrm{T}$ cell lymphoma they represent part of the neoplastic population. Moroch et al. [11] described five cases with initial diagnoses of lymphocyte-rich classical Hodgkin lymphoma, all of which were later reclassified as follicular $\mathrm{T}$ cell lymphoma after demonstration of T-follicular helper cell marker expression in the rosetting $\mathrm{T}$ cells. A systematic analysis of the immunophenotype of the rosetting $\mathrm{T}$ cells in follicular $\mathrm{T}$ cell lymphoma remains lacking, however.

The aim of the present study was to characterize the morphological features and immunophenotype of neoplastic rosetting $\mathrm{T}$ cells and Hodgkin-Reed-Sternberg-like cells in follicular $\mathrm{T}$ cell lymphoma, in order to define specific characteristics that could be helpful in the differential diagnosis of other malignant lymphomas and could provide new insights concerning the rosetting phenomenon of this tumor.

\section{Material and methods}

\section{Patients and immunostainings}

All cases diagnosed as follicular T cell lymphoma at the Dr. Senckenberg Institute of Pathology between 2010 and 2017 were retrieved from the archive. During the diagnostic workup, all lymph nodes were stained for CD20, CD3, PD1, CD23, CD10, BCL6, ICOS, CXCL13, MUM1, CD30, CD15, PAX5, and LMP1. Missing stainings were completed before the review. All cases were reviewed by the authors (CA, M-LH, SH, JM, AP, SAP, ES), and diagnoses of follicular $\mathrm{T}$ cell lymphoma were confirmed according to the 2017 revised edition of the WHO Classification of Tumors of Haematopoietic and Lymphoid Tissues. A subset of cases was additionally stained for IgD, CCL17, and CCR4. The local ethics committee of Frankfurt University Hospital agreed to the study. Antibodies, dilutions, and providers are listed in Suppl. Table S1. For cases that did not show any positive signals in LMP1 staining, additional Epstein-Barr encoding region (EBER) in situ hybridization was performed. Tfollicular helper cell markers and other markers expressed in the Hodgkin-Reed-Sternberg-like cells of follicular $\mathrm{T}$ cell lymphoma were scored positive when at least $60 \%$ of the cell population was positive. For the presence of rosetting $\mathrm{T}$ cells around Hodgkin-Reed-Sternberg-like cells, the presence of at least one complete rosette was assessed as positive. In all cases, immunoglobulin heavy chain gene rearrangements (PCRs for framework regions $\mathrm{I}-\mathrm{III}$ ) and $\mathrm{T}$ cell receptor gamma clonality tests (Invivoscribe Technologies, San Diego, CA, USA) were performed. For a subset of cases, additional clonality tests for $\mathrm{T}$ cell receptor beta and immunoglobulin kappa light chain were performed. 
Fig. 1 Typical morphologic and architectural features of follicular $\mathrm{T}$ cell lymphoma. a, b Neoplastic T-follicular helper cells appear as pale clear cells in the neighborhood of and rosetting around

Hodgkin-Reed-Sternberg-like cells (case $1, \mathbf{a}, \mathrm{HE} \times 40, \mathbf{b} \mathrm{HE}$ $\times 200)$.

c, $\mathbf{d}$ Follicular $\mathrm{T}$ cell lymphoma case 4 with neoplastic T-

follicular helper cells representing only a minority of the infiltrate. These are strongly highlighted by PD1 (c, PD1 immunostaining, $\times 40$ ) and appear as moth-eaten holes in the CD20 immunostaining (d, CD20 immunostaining, $\times 40$ )
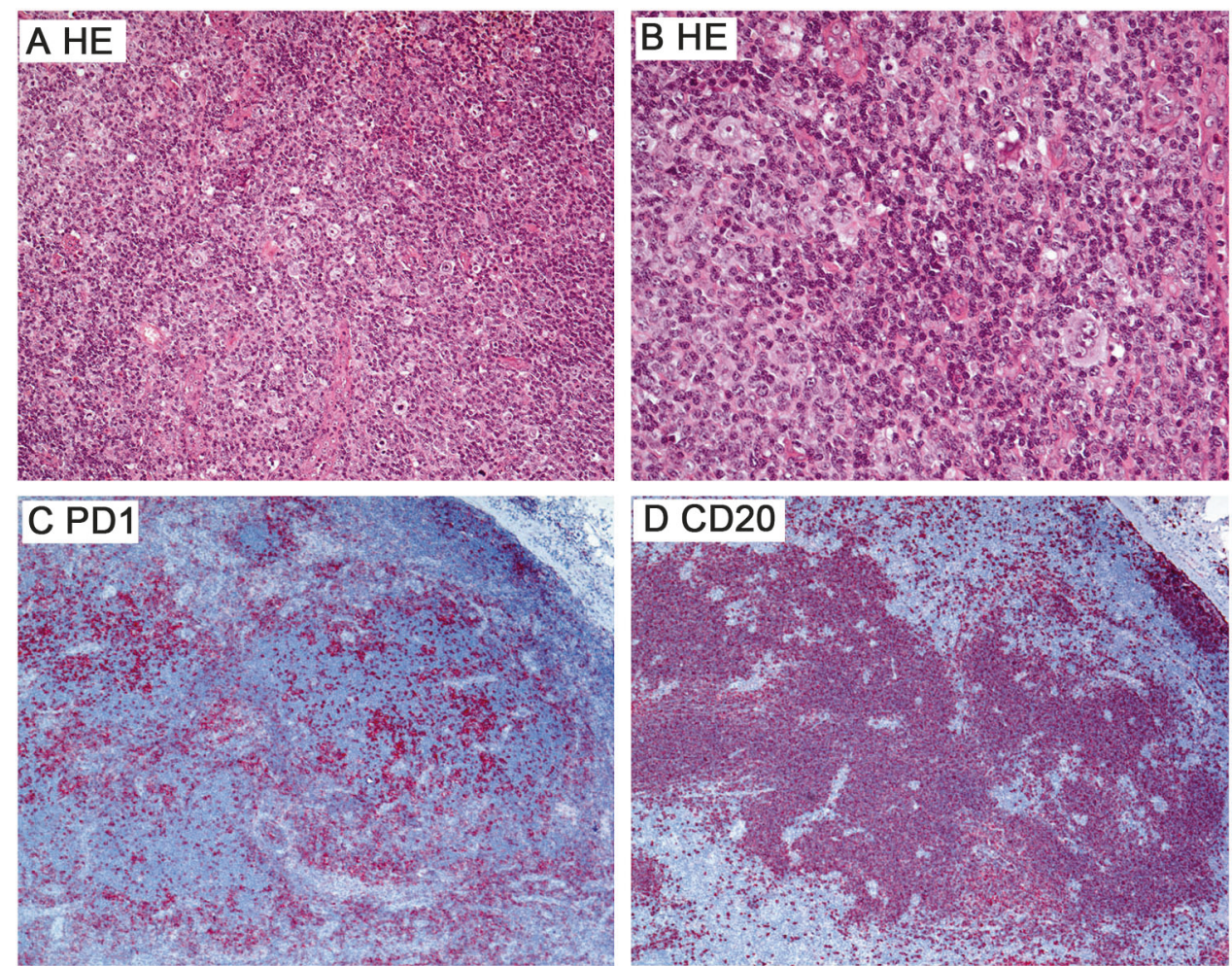

\section{Results}

\section{Patient characteristics}

Sixteen patients who had been diagnosed with follicular T cell lymphoma were identified. The median age was 66 years (range 49-89 years, Suppl. Table S2) and 10 of the 16 patients were males $(63 \%)$. The location of the lymph node biopsy was inguinal in $7 / 16$ cases $(44 \%)$, cervical in $5 / 16$ patients $(31 \%)$, and axillary in two patients (13\%). In the other two patients, the lymph node localization was unknown.

\section{Morphology and microenvironment}

Morphological patterns were evaluated in all cases. Only 2 cases showed a follicular lymphoma-like pattern, whereas the other 14 cases presented a progressively transformed germinal center-like pattern. All cases showed a more or less partial infiltrate of the lymph node, which in some cases consisted of only a minor population of neoplastic cells. Follicular dendritic cell meshworks were usually restricted to the areas of follicle remnants and no larger expansions of follicular dendritic cells were encountered. The neoplastic cells were usually recognized in the hematoxylin and eosin (H\&E) staining based on a pale appearance, with enlarged and activated nuclei, and often a clear cytoplasm (Fig. 1). Epithelioid histiocytes were present in $12 / 16$ cases $(75 \%)$. In the CD20 immunostaining, the infiltration pattern of neoplastic $\mathrm{T}$ cells was plainly visible, resulting in CD20-negative moth-eaten holes in or around remnants of B cell follicles. This pattern was reminiscent of activated $\mathrm{T}$ cells with clear cytoplasm, which can be observed in nodular lymphocyte predominant Hodgkin lymphoma (Fig. 1) [12]. Four of the sixteen cases presented an expanded population of CD23-positive mantle zone B cells around the neoplastic infiltrates (Fig. 2). In all 14 cases tested, an IgD-positive mantle zone B cell population was observed. In case 10, the mantle zone B cell population was so prominent that the T-follicular helper cell population with a complete T-follicular helper cell phenotype and rosetting around Hodgkin-Reed-Sternberg-like cells was difficult to perceive (Fig. 2). This patient had an original diagnosis of small lymphocytic lymphoma, and only when he relapsed after 2 years with an overt nodal T-follicular helper cell-related peripheral $\mathrm{T}$ cell lymphoma were both specimens sent for a second opinion, leading to the retrospective diagnosis of follicular T cell lymphoma. In this biopsy, a monoclonal $\mathrm{T}$ cell receptor gamma rearrangement was obtainedidentical to the monoclonal $\mathrm{T}$ cell receptor gamma rearrangement observed in the follow-up biopsy with overt Tfollicular helper cell lymphoma. The immunoglobulin heavy chain clonality test was polyclonal. We therefore considered this first biopsy to be a follicular T cell lymphoma, B cell-rich variant. 

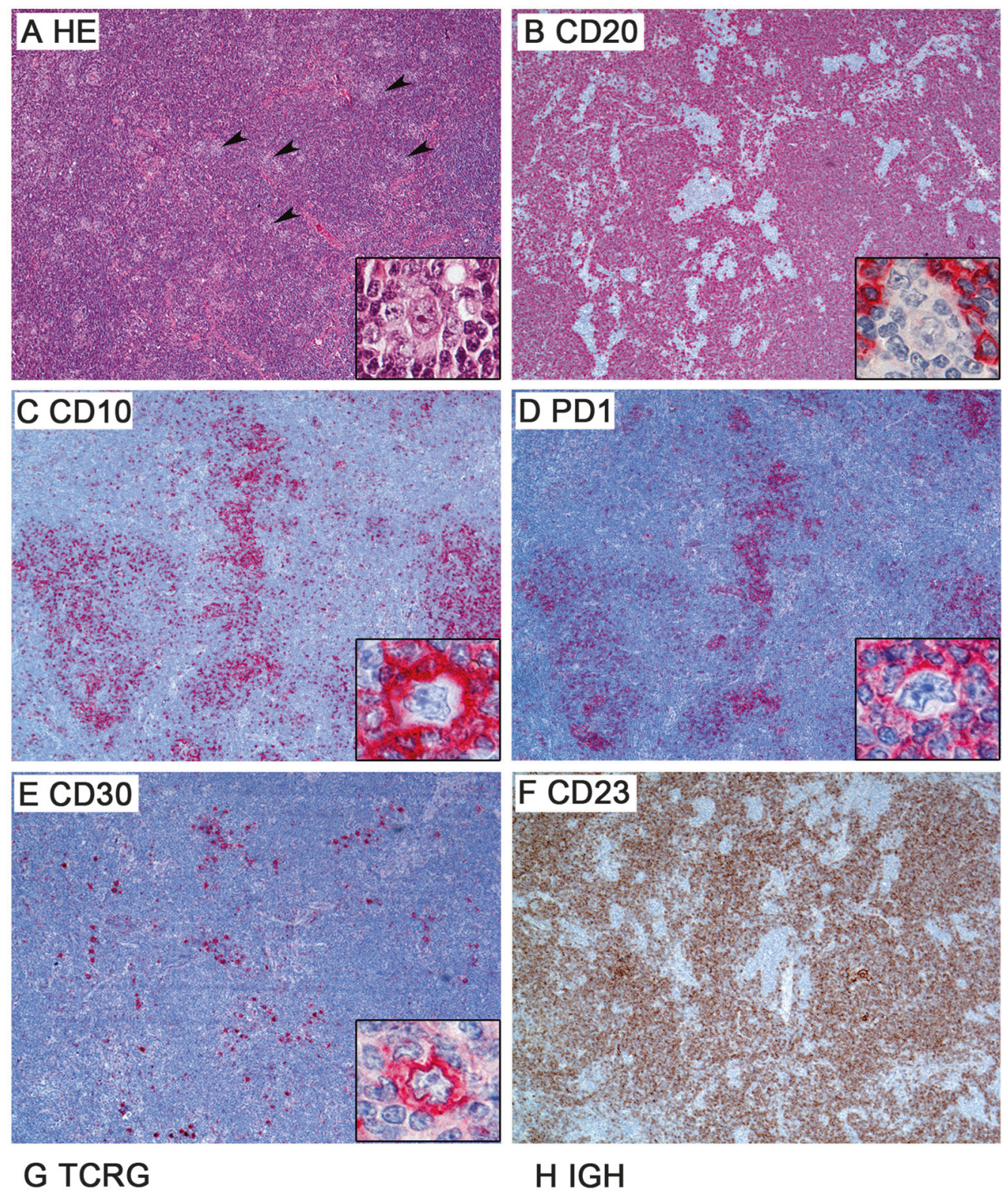

\section{$\mathrm{H}$ IGH}
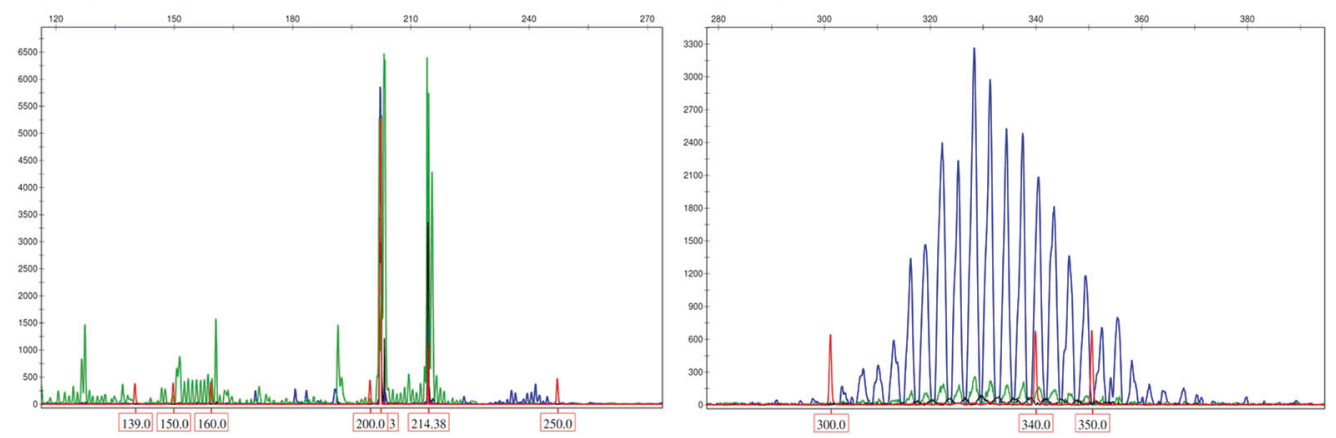
Fig. 2 B cell-rich variant of follicular T cell lymphoma. Case 10 was originally diagnosed as lymphocytic lymphoma, and only when the patient relapsed 2 years later with a nodal peripheral $\mathrm{T}$ cell lymphoma with $\mathrm{T}$-follicular helper cell phenotype, the diagnosis of follicular $\mathrm{T}$ cell lymphoma was retrospectively made, when both specimens were sent for a second opinion. a Infiltrate consisting mainly of small lymphoid cells with scattered more pale areas of T-follicular helper cells (arrowheads), originally mistaken as proliferation centers (HE, $\times 40)$. Inset $(\times 400)$ with an Hodgkin-Reed-Sternberg-like cells surrounded by activated lymphocytes, representing $\mathrm{T}$-follicular helper cells. b CD20 immunostaining highlighting the high number of B cells ( $\times 40)$ with negative areas of T-follicular helper cells. Inset with a CD20-negative Hodgkin-Reed-Sternberg-like cell surrounded by Tfollicular helper cells $(\times 400)$. c CD10 immunostaining, highlighting scattered aggregates of T-follicular helper cells $(\times 40)$, inset with a CD10-positive $T$ cell rosette $(\times 400)$. d PD1 immunostaining, highlighting scattered aggregates of T-follicular helper cells $(\times 40)$, inset with a PD1-positive $T$ cell rosette $(\times 400)$. e CD30 immunostaining highlighting the Hodgkin-Reed-Sternberg-like cells accumulated in the areas of T-follicular helper cells $(\times 40)$. Inset with a CD30-positive Hodgkin-Reed-Sternberg-like cell $(\times 400)$. f CD23-positive diffusely distributed (mantle zone) B cells with CD23-negative areas of Tfollicular helper cells. No CD23-positive follicular dendritic cell proliferation was observed. $\mathbf{g} \mathrm{T}$ cell receptor gamma clonality analysis demonstrated a reproducible biallelic rearrangement. h Immunoglobulin heavy chain clonality analysis showing a polyclonal result

\section{Immunoglobulin and $\mathbf{T}$ cell receptor gene rearrangement analysis}

$\mathrm{T}$ cell clonality analyses for $\mathrm{T}$ cell receptor gamma were performed in all cases and were technically evaluable in 15 / 16 biopsies. All evaluable cases showed a clonal $\mathrm{T}$ cell receptor gamma and/or beta rearrangement (monoallelic or bialellic). In two of these cases there was a strong admixed polyclonal background. Concerning immunoglobulin heavy chain gene rearrangements, four cases showed a clonal product with polyclonal background in one of the three immunoglobulin heavy chain framework regions, with the clonal peak not being detected in the other two framework regions. The remaining 11 cases presented a polyclonal immunoglobulin heavy chain clonality result. The four cases with clonal immunoglobulin heavy chain peaks did not differ from the 11 cases with polyclonal immunoglobulin heavy chain results in terms of morphology or immunophenotype of Hodgkin-Reed-Sternberg-like cells, or in terms of the abundance of reactive $B$ cells in the microenvironment.

\section{Immunophenotype of the neoplastic cells}

As required by the 2017 revised edition of the WHO Classification of Tumors of Haematopoietic and Lymphoid Tissues, the neoplastic cells in all cases expressed at least two T-follicular helper cell markers. Since many tumors show an intratumoral heterogeneity, the T-follicular helper cell immunophenotype was assessed for the neoplastic cell compartment in general and separately for the rosetting T cells around Hodgkin-Reed-Sternberg-like cells for every T-follicular helper cell staining. The bulk neoplastic cells were positive for PD1 and ICOS in all cases (Table 1 and Suppl. Table 2), and positive for CD10, BCL6, and CXCL13 in the majority of cases (Table 1). IRF4/MUM1 expression in the neoplastic population was less frequently found $(44 \%)$. Surprisingly, a high number of cases (12/16, $75 \%)$ presented at least a partial ( $>50 \%)$ expression of CD30 in the neoplastic cells. CD30 expression was typically less pronounced than in the Hodgkin-Reed-Sternberg-like cells, and usually membrane-bound and/or dot-like in the Golgi area (Fig. 3).

Neoplastic rosetting $\mathrm{T}$ cells around Hodgkin-Reed-Sternberg-like cells were found in all cases with Hodgkin-Reed-Sternberg-like cells (15/16 follicular T cell lymphoma cases, 94\%). These rosetting $\mathrm{T}$ cells were positive for PD1, ICOS, and CD10 in 87, 67, and 60\% of cases, respectively (Table 1 and Fig. 4). The expression of CXCL13, BCL6, or IRF4/MUM1 in rosetting neoplastic $\mathrm{T}$ cells was limited to a small fraction of cases (Table 1). When compared with 10 cases of lymphocyte-rich classical Hodgkin lymphoma, expression of CD10 in the rosetting $\mathrm{T}$ cells and CD30 in a subset of the neoplastic population in general were the best discriminating markers (Table 1).

Twelve follicular $\mathrm{T}$ cell lymphoma were stained for CCR4, which is usually expressed in normal T-helper type 2 and regulatory $\mathrm{T}$ cells [13]. Two of the 12 follicular T cell lymphoma presented a homogeneous strong CCR4 expression in the tumor cells, whereas the other 10 cases showed a heterogeneous and variable CCR4 expression in the neoplastic cells (Fig. 5).

\section{Immunophenotype of Hodgkin-Reed-Sternberg- like cells}

Because the diagnosis of follicular $\mathrm{T}$ cell lymphoma is frequently challenging and these cases can easily be misdiagnosed as classical Hodgkin lymphoma, we also evaluated the immunophenotype of the Hodgkin-Reed-Sternberg-like cells in order to find parameters helpful for distinguishing them from true Hodgkin-Reed-Sternberg cells in classical Hodgkin lymphoma. Hodgkin-Reed-Sternberg-like cells in all 15 cases with identifiable Hodgkin-Reed-Sternberg-like cells were positive for CD30 and IRF4/MUM1, typically strongly positive for PAX5 (Table 2 and Suppl. Figure 1) and, in the majority of cases (13/15 cases, $87 \%$ ), positive for EBV based on either LMP1 immunostaining or EBER in situ hybridization. CD20 was expressed at variable levels in 4/ 15 cases $(27 \%)$ and CD15 was rarely positive (4/15 cases, 27\%). Because Hodgkin-Reed-Sternberg cells in classical Hodgkin lymphoma are usually positive for TARC/CCL17, 
Table 1 Immunophenotype of bulk population of neoplastic $\mathrm{T}$ cells and of rosetting neoplastic $\mathrm{T}$ cells in follicular $\mathrm{T}$ cell lymphoma in comparison to rosetting $\mathrm{T}$ cells in classical Hodgkin lymphoma (lymphocyte-rich variant)

\begin{tabular}{llll}
\hline Antigen & $\begin{array}{l}\text { Bulk population of } \\
\text { neoplastic T cells } \\
\text { positive }\end{array}$ & $\begin{array}{l}\text { Rosetting neoplastic } \\
\text { T cells positive }\end{array}$ & $\begin{array}{l}\text { Rosetting T cells in classical } \\
\text { Hodgkin lymphoma lymphocyte- } \\
\text { rich variant positive }\end{array}$ \\
\hline CD10 & $14 / 16(88 \%)$ & $9 / 15(60 \%)$ & $0 / 11(0 \%)$ \\
PD1 & $16 / 16(100 \%)$ & $13 / 15(87 \%)$ & $7 / 11(64 \%)$ \\
ICOS & $15 / 15(100 \%)$ & $10 / 15(67 \%)$ & $5 / 11(45 \%)$ \\
CXCL13 & $12 / 16(75 \%)$ & $3 / 15(20 \%)$ & $1 / 11(9 \%)$ \\
BCL6 & $11 / 16(69 \%)$ & $2 / 15(13 \%)$ & $0 / 11(0 \%)$ \\
IRF4/ & $7 / 16(44 \%)$ & $4 / 15(25 \%)$ & $2 / 11(18 \%)$ \\
MUM1 & & & $0 / 11(0 \%)$ \\
CD30 & $12 / 16(75 \%)$ & $0 / 16(0 \%)$ & \\
\hline
\end{tabular}

we also tested the Hodgkin-Reed-Sternberg-like cells in follicular T cell lymphoma. All 12 follicular T cell lymphoma cases tested showed expression of CCL17 in the Hodgkin-Reed-Sternberg-like cells, as is observed for Hodgkin-Reed-Sternberg cells in classical Hodgkin lymphoma (Fig. 5). Thus, the immunophenotype of Hodgkin-Reed-Sternberg-like cells in follicular $\mathrm{T}$ cell lymphoma does not allow for a clear distinction from Hodgkin-Reed-Sternberg cells in classical Hodgkin lymphoma.

\section{Past medical history and follow-up of follicular T cell lymphoma patients}

Six of 16 follicular T cell lymphoma patients (38\%) presented a past medical history of lymphoma (Suppl. Table 2). In brief, four patients had a T-follicular helper cellassociated neoplasm before the diagnosis of follicular $\mathrm{T}$ cell lymphoma and two patients had a history of a clonally unrelated diffuse large B cell lymphoma. For all but one patient, the slides of previous diagnoses were reviewed to confirm each diagnosis and compare it with the follicular $\mathrm{T}$ cell lymphoma infiltrate. One diffuse large B cell lymphoma was EBV-associated and occurred 4 years before the diagnosis of follicular $\mathrm{T}$ cell lymphoma. The other diffuse large B cell lymphoma was diagnosed 15 years before the follicular T cell lymphoma, with an involvement of the gut. This case showed a centroblastic morphology (germinal center B cell-like type according to Hans classifier [14]) and was EBV-negative. In both cases of diffuse large B cell lymphoma, there was no evidence of a $\mathrm{T}$-follicular helper cell population on immunohistochemistry, nor of a clonal amplificate in the $\mathrm{T}$ cell receptor gamma clonality analysis. The other four patients had a history of T-follicular helper cell-associated lymphomas; two of these patients had suffered from an early angioimmunoblastic T cell lymphoma 6 and 10 years before the diagnosis of follicular $\mathrm{T}$ cell lymphoma. In another of these patients, a follicular $\mathrm{T}$ cell lymphoma (slides of this case are unavailable) was diagnosed 7 years before the relapse of follicular $\mathrm{T}$ cell lymphoma. The fourth patient had a history of cutaneous B cell lymphoma 5 years before the diagnosis of follicular $\mathrm{T}$ cell lymphoma (case 14). At revision of this diagnosis, a prominent T-follicular helper cell population was observed rosetting around single EBV-infected Hodgkin-ReedSternberg-like cells, together with the clonal $\mathrm{T}$ cell receptor gamma rearrangement, suggesting an early state of evolving follicular $\mathrm{T}$ cell lymphoma (Fig. 6). Interestingly, in this case, the clonal peak in the $\mathrm{T}$ cell receptor gamma rearrangement was not identical to the clonal rearrangement in the follow-up biopsy of overt follicular $\mathrm{T}$ cell lymphoma, thus raising the possibility of a systemic and possibly oligoclonal T-follicular helper cell expansion. Both clonality assays for immunoglobulin heavy and kappa light chain gene rearrangements were polyclonal in this infiltrate, and the expanded B cell population was IgD-positive. The $\mathrm{B}$ cell population in this case therefore likely presented an expanded mantle zone B cell population, as observed in case 10 .

Relapses after the diagnosis of follicular $\mathrm{T}$ cell lymphoma occurred in five patients (31\%). In two patients, relapses occurred after 6 months as angioimmunoblastic $T$ cell lymphoma and T-follicular helper cell-related peripheral $\mathrm{T}$ cell lymphoma. The other three patients presented relapses of nodal $\mathrm{T}$-follicular helper cell-related peripheral $\mathrm{T}$ cell lymphoma 1 and 2 years after the diagnosis of follicular $\mathrm{T}$ cell lymphoma.

In summary, a group of the patients showed relatively rapid progress to an aggressive peripheral $\mathrm{T}$ cell lymphoma, whereas other cases relapsed as follicular $\mathrm{T}$ cell lymphoma after 6-10 years, pointing to a rather indolent behavior. Treatment and follow-up times were heterogeneous, however, and therefore no consistent conclusions can be drawn from these data.

\section{Discussion}

In the present study, we analyzed a relatively large number of follicular $\mathrm{T}$ cell lymphoma cases, in regard to the 
Fig. 3 CD30 expression in Hodgkin-Reed-Sternberg-like and neoplastic T-follicular helper cells in follicular $\mathrm{T}$ cell lymphoma. a, b. Case 14 showing strongly CD30positive

Hodgkin-Reed-Sternberg-like cells (highlighted by arrows) and in the neighborhood several more faintly positive CD30positive neoplastic cells (highlighted by arrowheads), a few of them with close contact to the

Hodgkin-Reed-Sternberg-like cells.

a CD30 immunostaining, $\times 40$ and b CD30 immunostaining, $\times 400$. c, $\mathbf{d}$ Case 1 showing strongly CD30-positive Hodgkin-Reed-Sternberg-like cells (examples highlighted by arrows) and in the neighborhood several more faintly positive CD30-positive neoplastic cells (highlighted by arrowheads). c CD30 immunostaining, $\times 40$ and d CD30 immunostaining, $\times 400$. e, $\mathbf{f}$ Typical classical Hodgkin lymphoma (mixed cellularity type) with CD30 expression in

Hodgkin-Reed-Sternberg cells but CD30-negative surrounding $\mathrm{T}$ cells.

e CD30 immunostaining, $\times 200$ and $\mathbf{f}$ CD30 immunostaining, $\times 400$
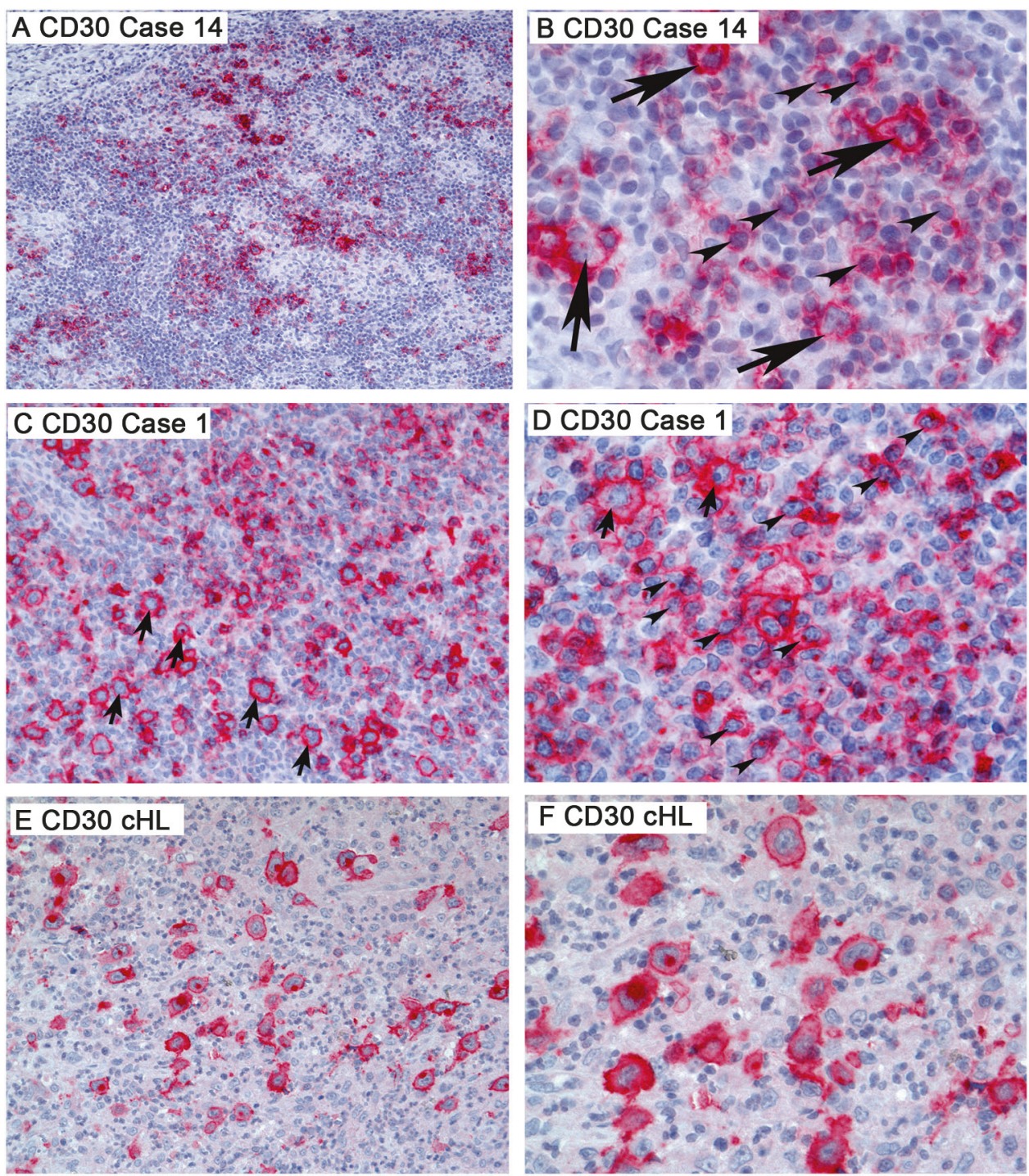

rareness of the disease. The involvement of mainly elderly patients with a slight male predominance fits with the data of previous studies [3, 15]. In the present series, however, there was a relatively high number of inguinal biopsies, for which we do not have an explanation.

Hodgkin-Reed-Sternberg-like cells were found in most cases, emphasizing the importance of classical and nodular lymphocyte predominant Hodgkin lymphoma as differential diagnoses [11]. In the present study, Hodgkin-Reed-Sternberg-like cells were CD20-positive in $27 \%$ of cases and, in most instances, were EBV-positive, which is in line with previous studies [3, 11, 16]. CD15 was less frequently expressed by the Hodgkin-Reed-Sternberg-like cells in our study in comparison to the findings of a previous study [16], which is likely related to the different antibody clones applied in the two studies. CCL17 was positive both in typical Hodgkin-Reed-Sternberg cells in classical Hodgkin lymphoma and in Hodgkin-Reed-Sternberg-like cells in follicular T cell lymphoma; therefore, CCL17 does not aid in differentiating between the two lymphoma types. Generally, there was no specific immune marker in the immune phenotype of Hodgkin-Reed-Sternberg-like cells that allowed a clear differentiation from Hodgkin-Reed-Sternberg cells in classical Hodgkin lymphoma. Particularly, lymphocyte-rich classical Hodgkin lymphoma is an important differential diagnosis to follicular $\mathrm{T}$ cell lymphoma because it also presents a B cell-rich microenvironment, CD20 positivity of Hodgkin-Reed-Sternberg cells in $32 \%$ of the cases and a high number of cases with EBV-infected Hodgkin-ReedSternberg cells (47\%) [17]. Both lymphoma types frequently present a nodular growth pattern; however, lymphocyte-rich classical Hodgkin lymphoma is more frequently found in the Waldeyer's ring and in cervical lymph nodes, in contrast to the frequent inguinal localization of follicular $\mathrm{T}$ cell lymphoma. In middle-aged to elderly persons with inguinal lymph node biopsies and CD20-positive and EBV-positive 
Fig. 4 Immunophenotype of neoplastic rosetting T-follicular helper cells in follicular $\mathrm{T}$ cell lymphoma. Case 1 presents rosetting T-follicular helper cells positive for PD1 (a, ×200), ICOS $(\mathbf{b}, \times 400)$, CXCL13 (c, $\times 200)$, CD10 (d, $\times 400)$, BCL6 $(\mathbf{e}, \times 400)$, and IRF4/ MUM1 $(\mathbf{f}, \times 400)$
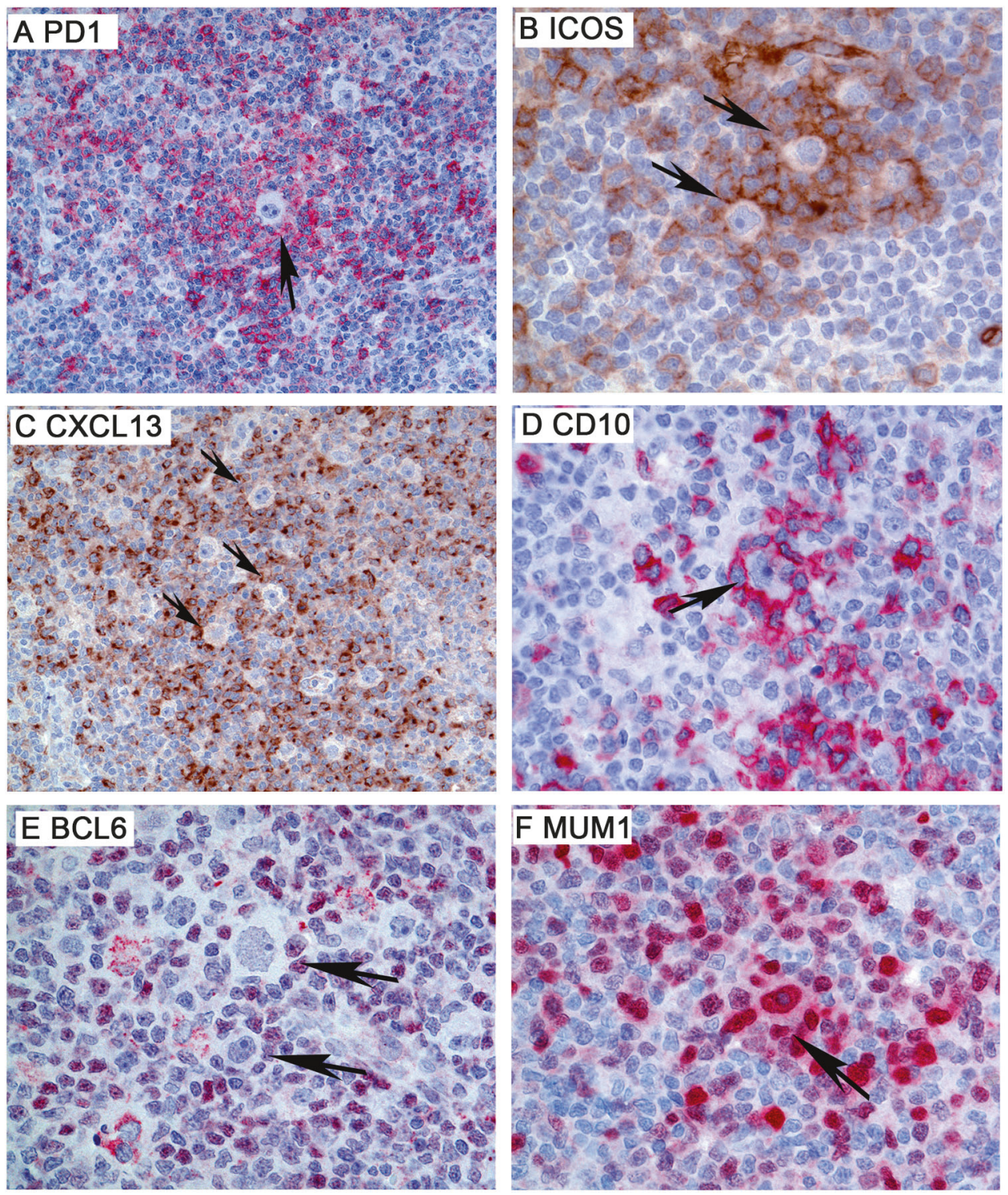

Hodgkin-Reed-Sternberg(-like) cells in a rather B cell-rich background, the exclusion of a follicular $\mathrm{T}$ cell lymphoma with Hodgkin-Reed-Sternberg-like cells should be mandatory. Follicular $\mathrm{T}$ cell lymphoma cases in the present study mimicked not only Hodgkin lymphoma, but also small lymphocytic lymphoma or cutaneous B cell lymphoma. Furthermore, in small lymphocytic lymphoma/chronic lymphatic leukemia, Hodgkin-Reed-Sternberg-like cells are occasionally seen, and these are often EBV-positive and unrelated to the lymphoma clone [18]. In this study, none of the cases with follow-up available relapsed as classical or nodular lymphocyte predominant Hodgkin lymphoma or diffuse large B cell lymphoma, indicating how difficult the diagnosis of this entity can be. B cell-rich variants of this entity have been described before [3].

The presence of Hodgkin-Reed-Sternberg-like cells may be important not only from the diagnostic point of view, but may also give hints to the pathophysiology of the development of follicular $\mathrm{T}$ cell lymphoma. In most cases investigated in this study, Hodgkin-Reed-Sternberg-like cells were EBV-infected. These cells can benefit from a proliferative advantage in the immunocompromised background of follicular T cell lymphoma. Conversely, neoplastic T-follicular helper cells may profit from the interaction with Hodgkin-Reed-Sternberg-like cells, as they recapitulate the interaction between B cells and T-follicular helper cells in the germinal center and thus provide constant active $\mathrm{T}$ cell receptor signaling, which transmits survival signals to the neoplastic T-follicular helper cell population. Activating mutations in the $\mathrm{T}$ cell receptor signaling pathway have been observed in T-follicular helper cell-derived lymphomas and may replace the activating stimulus by Hodgkin-ReedSternberg-like cells when the lymphoma progresses [19]. Follicular $\mathrm{T}$ cell lymphoma may represent an early type of 
Fig. 5 CCL17 and CCR4 expression in follicular T cell lymphoma. a Strong CCL17 expression in

Hodgkin-Reed-Sternberg cells of classical Hodgkin lymphoma and diffusion of CCL17 to the interstitium $(\times 200)$. b Moderate to strong CCL17 expression in Hodgkin-Reed-Sternberg-like cells of follicular $\mathrm{T}$ cell lymphoma (Case $8, \times 400)$. c Homogeneous CCR4 expression in T-follicular helper cells of follicular $\mathrm{T}$ cell lymphoma Case $8(\times 400)$, Hodgkin-Reed-Sternberg-like cells are highlighted by arrows. d Variable and heterogeneous CCR4 expression in T-follicular helper cells of Case $10(\times 400)$, Hodgkin-Reed-Sternberg-like cells are highlighted by arrows
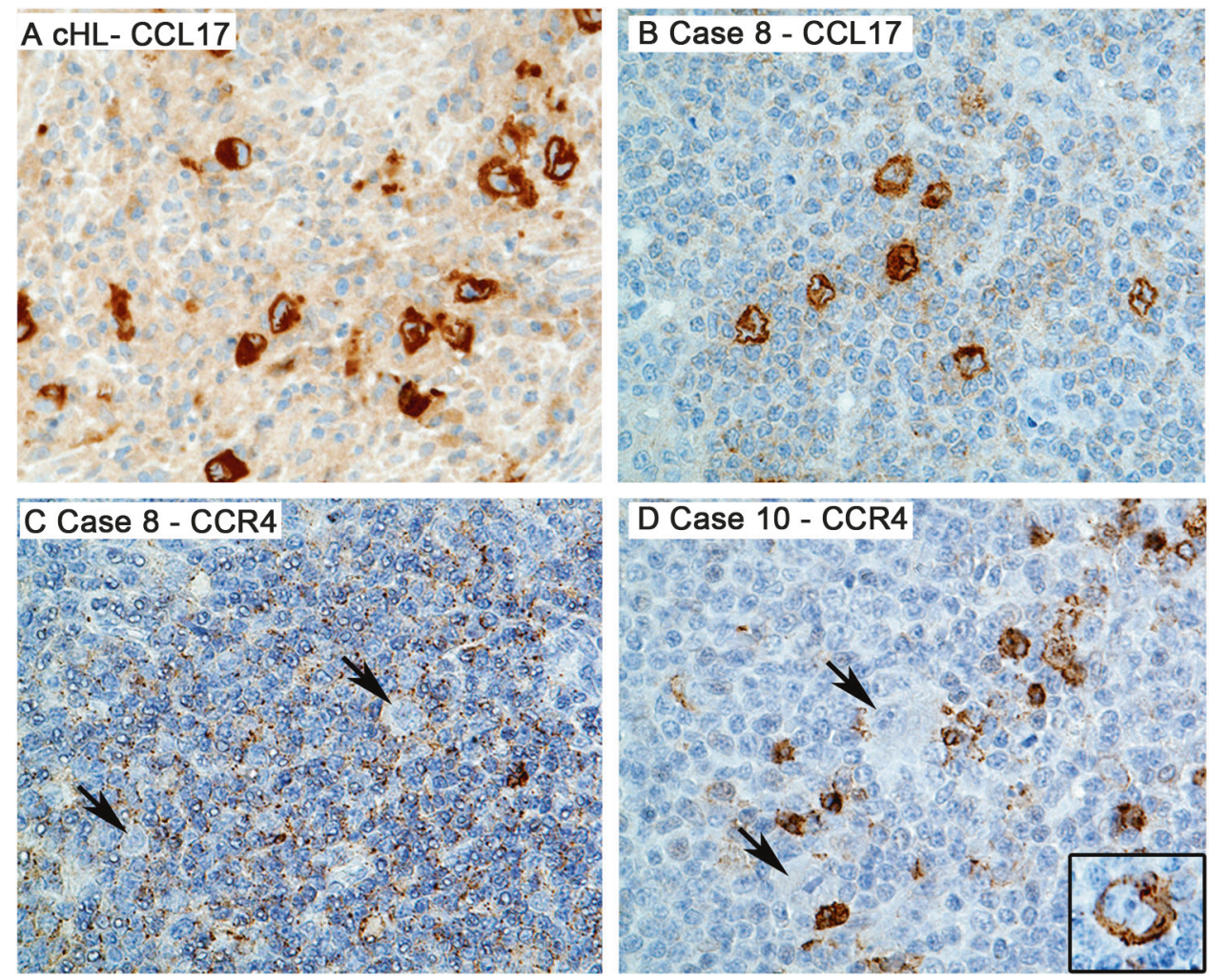

Table 2 Immunophenotype of Hodgkin-Reed-Sternberg-like cells in follicular T cell lymphoma

\begin{tabular}{ll}
\hline Antigen & $\begin{array}{l}\text { Hodgkin-Reed-Sternberg-like cells } \\
\text { positive }\end{array}$ \\
\hline CD20 & $4 / 15(27 \%)$ \\
CD30 & $15 / 15(100 \%)$ \\
CD15 & $4 / 15(27 \%)$ \\
LMP1 & $7 / 15(47 \%)$ \\
EBV (LMP1 and/or & $13 / 15(87 \%)$ \\
EBERbpositive) & \\
PAX5 & $15 / 15(100 \%)$ \\
IRF4/MUM1 & $15 / 15(100 \%)$ \\
\hline
\end{tabular}

T-follicular helper cell-derived lymphoma because the follicular architecture reminiscent of germinal centers is at least partly preserved and the neoplastic cells frequently only represent a minor population in the infiltrate. Comprehensive molecular studies of this entity are currently lacking, however, and thus their relationships with angioimmunoblastic $\mathrm{T}$ cell lymphoma and other $\mathrm{T}$-follicular helper cell-derived lymphomas can only be speculated upon. Because we observed expression of CCL17 in the Hodgkin-ReedSternberg-like cells in all cases tested, the Hodgkin-ReedSternberg-like cells likely attract the neoplastic T-follicular helper cells, which express CCR4 at variable levels.

PD1 and ICOS were positive in neoplastic cells in all cases, and CD10 was positive in the majority of cases, as also observed in previous studies [3, 11]. The discrepancy between the immunophenotype of bulk neoplastic cells and rosetting $\mathrm{T}$ cells was surprising, which raises the question of whether or not they represent varying differentiation stages or an up-anddown regulation of $\mathrm{T}$-follicular helper cell antigens depending on the localization in the tissue or interaction with other cell types, such as Hodgkin-Reed-Sternberg-like cells. When compared with the rosetting $\mathrm{T}$ cells in the microenvironment of lymphocyte-rich classical Hodgkin lymphoma, CD10 was the most specific marker to allow discrimination between these two lymphomas, as it was never expressed in the rosetting T cells of lymphocyte-rich classical Hodgkin lymphoma. All other T-follicular helper cell markers can be positive in both lymphoma types, although at a lower frequency in lymphocyte-rich classical Hodgkin lymphoma. The detection of a $\mathrm{T}$ cell clone, together with $\mathrm{CD} 10$ and CD30 expression in the neoplastic compartment, were the most reliable features for establishing a diagnosis of follicular $\mathrm{T}$ cell lymphoma. Fortunately, CD30 immunostaining is usually performed in the diagnostic work-up of all classical Hodgkin lymphoma biopsies, and thus expression in the neoplastic $\mathrm{T}$ cells in a subset of cases can be an important diagnostic clue. CD30 expression in the neoplastic population in a subset of follicular $\mathrm{T}$ cell lymphoma cases likely reflects the activation of these neoplastic cells, possibly through stimulation via the Hodgkin-Reed-Sternberg-like cells.

To enable more comprehensive clinical and molecular studies of follicular $\mathrm{T}$ cell lymphoma, it is first important 
Fig. 6 B cell-rich cutaneous Tfollicular helper cell lymphoma with tumor cells rosetting around EBV-infected

Hodgkin-Reed-Sternberg-like cells. This cutaneous biopsy showing a B cell-rich infiltrate with aggregates of T-follicular helper cells was taken 5 years before the diagnosis of a nodal follicular T cell lymphoma in an inguinal lymph node (Case 14). a, b HE stainings showing an infiltrate mainly composed by small-sized to medium-sized lymphocytes, some with a more pale cytoplasm and some blastoid B cells (arrowheads, b) (a, $\times 40$ and b, $\times 200)$. c CD20 immunostaining demonstrating that the majority of the infiltrate is composed by B cells; however, T-follicular helper cells appear as moth-eaten areas (arrows, $\times 100)$. Inset $(\times 200)$ is displaying such a T-follicular helper cell area in higher magnification. d CD3 immunostaining highlighting $\mathrm{T}$ follicular helper cells rosetting around an EBV-infected blastoid $B$ cell (arrows, $\times 200$ ).

e Rosetting T-follicular helper cells are positive for PD1 (arrows, $\times 200$ ). f Rosetting Tfollicular helper cells are positive for CD10 (arrows, $\times 200)$. g Rosetting T-follicular helper cells are positive for ICOS (arrows, ×200). h EBER in situ hybridization demonstrating EBV-infected blastoid B cells $(\times 100)$
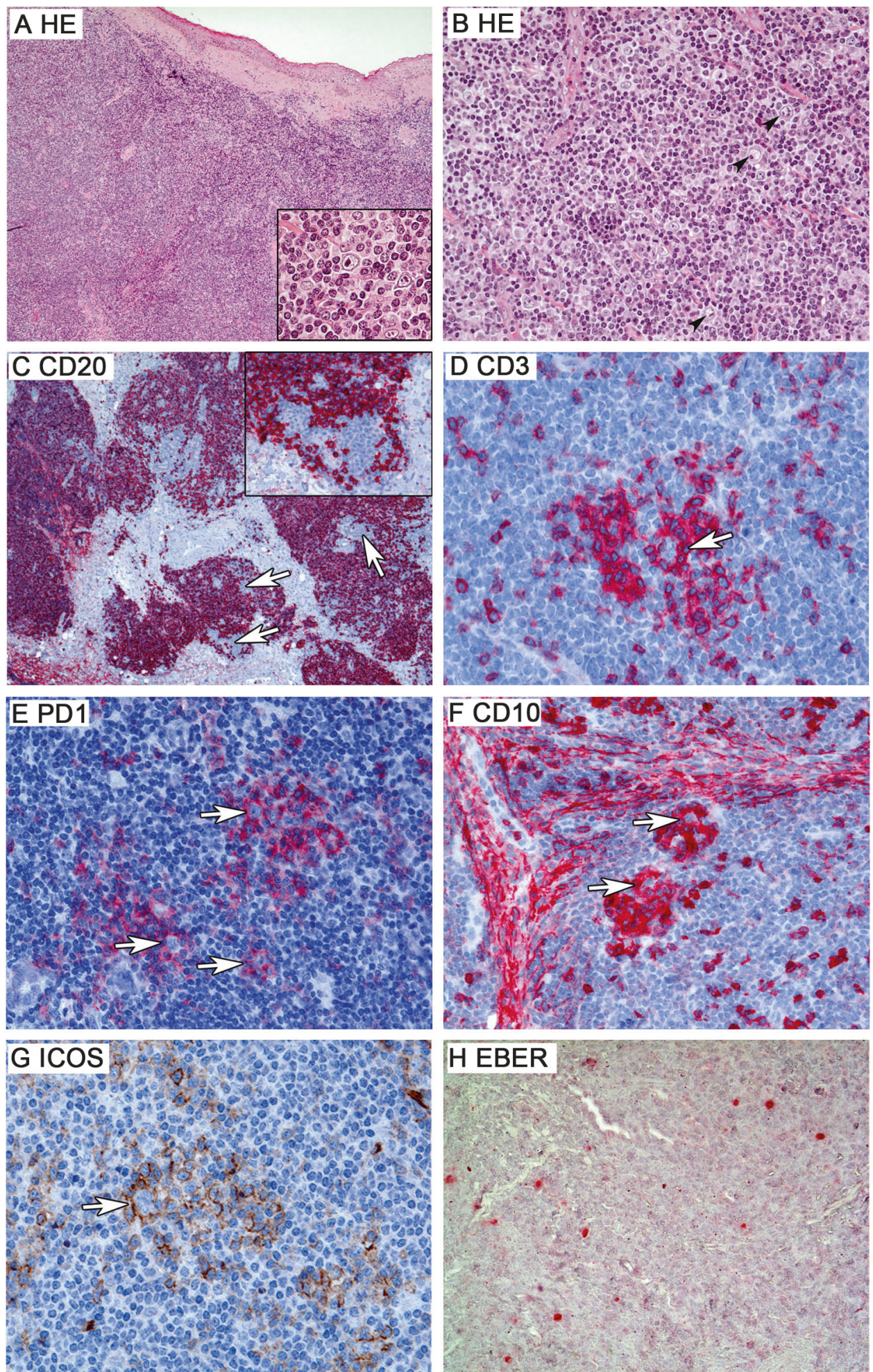

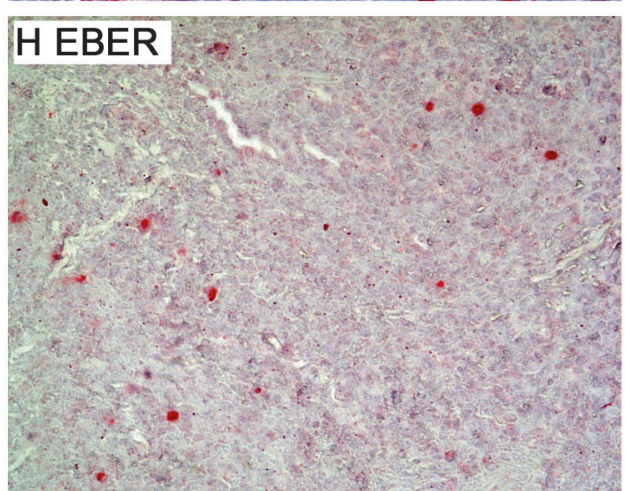

that these cases are adequately recognized. Because Hodgkin-Reed-Sternberg-like cells in follicular $\mathrm{T}$ cell lymphoma may be indistinguishable from Hodgkin-ReedSternberg cells in classical Hodgkin lymphoma, it is important to identify the neoplastic clone in the environment of these cells. In this regard, CD30 expression by neoplastic $\mathrm{T}$ cells can represent a useful diagnostic tool, in addition to being a potential therapeutic target. The collection of larger series of well-documented cases can prospectively expand our knowledge concerning the mutational 
landscape of follicular $\mathrm{T}$ cell lymphoma to determine whether it represents a prodromal phase of a full-blown angioimmunoblastic $\mathrm{T}$ cell lymphoma or another nodal $\mathrm{T}$ follicular helper cell-related peripheral $\mathrm{T}$ cell lymphoma.

Acknowledgements This work was supported by the Deutsche Forschungsgemeinschaft (grants HA6145/2-1, KU1315/9-2; FOR 1961). We thank Yvonne Michel, Monica Mörtel, Elena Hartung, Susanne Hansen, and Lukas Oettler for their excellent technical assistance.

\section{Compliance with ethical standards}

Conflict of interest The authors declare that they have no conflict of interest.

\section{References}

1. Swerdlow SH, Campo E, Harris NL, Jaffe ES, Pileri SA, Stein H, et al. WHO Classification of Tumours of Haematopietic and Lymphoid Tissues: International Agency for Research on Cancer; Angioimmunoblastic T-cell lymphoma and other nodal lymphomas of $\mathrm{T}$ follicular helper (TFH) cell origin. 2017. p. 407-12. International Agency for Research on Cancer, Lyon 2017

2. Dobay MP, Lemonnier F, Missiaglia E, Bastard C, Vallois D, Jais JP et al. Integrative clinicopathological and molecular analyses of angioimmunoblastic T-cell lymphoma and other nodal lymphomas of follicular helper T-cell origin. Haematologica. 2017;102: e148-51.

3. Huang Y, Moreau A, Dupuis J, Streubel B, Petit B, Le Gouill S, et al. Peripheral T-cell lymphomas with a follicular growth pattern are derived from follicular helper T cells (TFH) and may show overlapping features with angioimmunoblastic T-cell lymphomas. Am J Surg Pathol. 2009;32:682-90.

4. Streubel B, Vinatzer U, Willheim M, Raderer M, Chott A. Novel t $(5 ; 9)(\mathrm{q} 33 ; \mathrm{q} 22)$ fuses ITK to SYK in unspecified peripheral T-cell lymphoma. Leukemia. 2006;20:313-8.

5. Attygalle AD, Feldman AL, Dogan A. ITK/SYK translocation in angioimmunoblastic T-cell lymphoma. Am J Surg Pathol. 2013;37:1456-7.

6. Quintanilla-Martinez L, Fend F, Moguel LR, Spilove L, Beaty MW, Kingma DW, et al. Peripheral T-cell lymphoma with Reed-Sternberg-like cells of B-cell phenotype and genotype associated with Epstein-Barr virus infection. Am J Surg Pathol. 1999;23:1233-40.

7. Bräuninger A, Spieker T, Willenbrock K, Gaulard P, Wacker HH, Rajewsky K, et al. Survival and clonal expansion of mutating "forbidden" (immunoglobulin receptor-deficient) Epstein-Barr virus-infected $\mathrm{b}$ cells in angioimmunoblastic $\mathrm{T}$ cell lymphoma. $\mathrm{J}$ Exp Med. 2001;194:927-40.
8. Mathas S, Hartmann S, Kuppers R. Hodgkin lymphoma: pathology and biology. Semin Hematol. 2016;53:139-47.

9. de Leval L, Savilo E, Longtine J, Ferry JA, Harris NL. Peripheral T-cell lymphoma with follicular involvement and a CD4+/bcl-6 +phenotype. Am J Surg Pathol. 2001;25:395-400.

10. Roers A, Montesinos-Rongen M, Hansmann ML, Rajewsky K, Küppers R. Amplification of TCRbeta gene rearrangements from micromanipulated single cells: $\mathrm{T}$ cells rosetting around Hodgkin and Reed-Sternberg cells in Hodgkin's disease are polyclonal. Eur J Immunol. 1998;28:2424-31.

11. Moroch J, Copie-Bergman C, de Leval L, Plonquet A, MartinGarcia N, Delfau-Larue MH, et al. Follicular peripheral T-cell lymphoma expands the spectrum of classical Hodgkin lymphoma mimics. Am J Surg Pathol. 2012;36:1636-46.

12. Nathwani B, Vornanen M, Winkelmann R, Kansal R, Döring C, Hartmann $S$, et al. Intranodular clusters of activated cells with $\mathrm{T}$ follicular helper (TFH) phenotype in nodular lymphocyte predominant Hodgkin lymphoma: a pilot study of 32 cases from Finland. Hum Pathol. 2013;44:1737-46.

13. Viola A, Luster AD. Chemokines and their receptors: drug targets in immunity and inflammation. Annu Rev Pharmacol Toxicol. 2008;48:171-97.

14. Hans CP, Weisenburger DD, Greiner TC, Gascoyne RD, Delabie $\mathrm{J}$, Ott $\mathrm{G}$, et al. Confirmation of the molecular classification of diffuse large B-cell lymphoma by immunohistochemistry using a tissue microarray. Blood. 2004;103:275-82.

15. Hu S, Young KH, Konoplev SN, Medeiros LJ. Follicular T-cell lymphoma: a member of an emerging family of follicular helper T-cell derived T-cell lymphomas. Hum Pathol. 2012;43:1789-98.

16. Nicolae A, Pittaluga S, Venkataraman G, Vijnovich-Baron A, Xi L, Raffeld M, et al. Peripheral T-cell lymphomas of follicular Thelper cell derivation with Hodgkin/Reed-Sternberg cells of Bcell lineage: both EBV-positive and EBV-negative variants exist. Am J Surg Pathol. 2013;37:816-26.

17. Anagnostopoulos I, Hansmann ML, Franssila K, Harris NL, Jaffe ES, Han J, et al. European Task Force on Lymphoma project on lymphocyte predominance Hodgkin disease: histologic and immunohistologic analysis of submitted cases reveals 2 types of Hodgkin disease with a nodular growth pattern and abundant lymphocytes. Blood. 2000;96:1889-99.

18. Kanzler H, Kuppers R, Helmes S, Wacker HH, Chott A, Hansmann ML, et al. Hodgkin and Reed-Sternberg-like cells in B-cell chronic lymphocytic leukemia represent the outgrowth of single germinal-center B-cell-derived clones: potential precursors of Hodgkin and Reed-Sternberg cells in Hodgkin's disease. Blood. 2000;95:1023-31.

19. Vallois D, Dobay MP, Morin RD, Lemonnier F, Missiaglia E, Juilland $\mathrm{M}$, et al. Activating mutations in genes related to TCR signaling in angioimmunoblastic and other follicular helper T-cellderived lymphomas. Blood. 2016;1128:1490-502. 